\title{
Drug Manufacturer
}

National Cancer Institute

\section{Source}

National Cancer Institute. Drug Manufacturer. NCI Thesaurus. Code C156626.

A person or entity that is engaged in the manufacturing, preparing, propagating,

compounding, processing, packaging, repackaging, or labeling of a prescription drug. 